\title{
Functional outcome of comminuted upper metaphyseal tibial fracture treated with circular external fixators
}

\author{
Parth Patel*, Harpreet Singh, Kamal Kumar Agarwal, Tilak Patel, Krushna Saoji, Dhruv Patel
}

Department of Orthopaedics, Geetanjali Medical College and Hospital, Udaipur, Rajasthan, India

Received: 15 February 2021

Revised: 22 March 2021

Accepted: 23 March 2021

\author{
*Correspondence: \\ Dr. Parth Patel, \\ E-mail: parthpate16435@gmail.com
}

Copyright: () the author(s), publisher and licensee Medip Academy. This is an open-access article distributed under the terms of the Creative Commons Attribution Non-Commercial License, which permits unrestricted non-commercial use, distribution, and reproduction in any medium, provided the original work is properly cited.

\begin{abstract}
Background: High speed motor vehicle accidents are on the rise over the past few years. These are responsible for most of the complex fractures of the tibial metaphysic. Proper treatment of these fractures is of paramount importance. The use of circular fixators for the definitive treatment of such fractures entails a minimally invasive technique of insertion that gives good fracture reduction and stability combined with minimal postoperative complications.

Methods: This study was prospective and conducted over 22 patients diagnosed with upper comminuted metaphyseal tibial fracture treated with circular external fixator.

Results: In this series, all 22 patients (100\%) were male. Fourteen cases (64\%) involved the left tibia and eight (36\%) fractures were on right. The mean patient age was 49.5 years (range 26-75). Road traffic collisions accounted for most of the injuries $(n=14)$, while fall from bike $(n=8)$. In this study, using knee society score evaluation, excellent results $(\mathrm{KSS}>80)$ were seen in 8 patients $(36.36 \%)$, good results (KSS 70-79) were seen in 10 patients $(45.45 \%)$, fair (KSS 60$69)$ in 4 patient $(18.18 \%)$ and poor $(\mathrm{KSS}<60)$ in 0 patient $(0 \%)$. Complication seen was pin tract infection in 2 patients (9.9\%), which was managed by regular pin site dressings. Another complication was non-union in 1 patient (4.54\%). No other complication was encountered.

Conclusions: Circular external fixation is a promising modality for surgical treatment of comminuted upper metaphyseal tibial fractures. It allows satisfactory realignment of the fractured fragments and their stabilization, besides early mobilization of the joints and care of associated soft tissue injuries, without significant complications.
\end{abstract}

Keywords: JESS, Hybrid, Ilizarov, Tibial metaphysis

\section{INTRODUCTION}

High speed motor vehicle accidents are on the rise over the past few years. These are responsible for most of the complex fractures of the tibialmetaphysis. ${ }^{1,2}$ Metaphyseal fractures of proximal tibia usually occur secondary to direct bending forces applied to the metaphyseal region of upper leg.

Proper treatment of these fractures is of paramount importance. Among the treatment of tibial fractures, the treatment of metaphyseal fractures of tibia remains challenging because of the sagittal and coronal malalignment which is mainly due to the mismatch between medullary canal diameter between the two fragments of the metaphyseal tibial fractures and anatomy of the metaphyseal region. Establishment of length and prevention of the coronal, sagittal, rotational malalignment is most important, during fixation. ${ }^{3}$

The choice of treatment depends upon the patient's age, his medical condition, the fracture configuration, degree of fracture displacement and condition of the overlying soft tissue cover, associated bony injuries and any neurovascular complications. The conservative management with cast or cast brace immobilization offers benefits of 
shorter hospital stay but it has a high level of complications. The use of internal fixation offers anatomic realignment and stability. In a compromised soft tissue condition, which is frequently associated with such fractures, this leads to further insult to the overlying tissues and can cause complications like infection, skin necrosis and exposure of hardware. ${ }^{4}$ With use of a minimally invasive technique, employing an external fixation it is possible to treat the patients with an immediate one stage procedure. ${ }^{5,6}$ Since reduction is less invasive, there is minimal soft-tissue exposure and blood loss. If needed, these fixators also allow for adjustment of the alignment and for compression/distraction both during and after surgery. Along with rigid fixation, it allows immediate mobilization of the joints and early weight bearing. ${ }^{7-11}$ The minimally invasive stabilization and reduction of comminuted fractures using distraction by external fixators employs the principle of ligamentotaxis.

This study has thus been conceptualized to study the functional outcome of the patients with high energy patterns of comminuted upper metaphyseal tibial injury treated with circular external fixators like Ilizarov, JESS and hybrid external fixator.

\section{Aim}

Aim of the study was to evaluate the functional outcome of comminuted upper metaphyseal tibial fracture treated with circular external fixators.

\section{Primary objective}

The study was done to evaluate the functional outcome of comminuted upper metaphyseal tibial fractures treated with circular external fixators using knee society score scoring system at 6 weeks, 3 months and 6 months.

\section{Secondary objective}

The secondary objective of the study was to analyse the result of external fixation with respect to bony union, soft tissue coverage, knee and ankle movement, limb shortening, duration of post-operative immobilization required and to assess the intra operative and postoperative complications.

\section{METHODS}

This study was prospective, observational and conducted over 22 patients diagnosed with upper comminuted metaphyseal tibial fracture coming to the department of orthopaedics of a tertiary care centre in southern Rajasthan between January 2019 to June 2020.

\section{Inclusion criteria}

Patients were included with upper metaphyseal tibial fracture with or without tibial plateau fracture, patient's age was over 18 year old, was able to work without assistance before injury and those who had given informed consent.

\section{Exclusion criteria}

Patients with distal neurovascular deficit of injured limb, polytrauma patients with other injuries of ipsilateral lower limb, patients with neoplastic condition and bilateral upper metaphyseal tibial fracture were excluded.

\section{Proposed statistical analysis}

Data entry was done with MS Excel windows 10. The descriptive analysis mean, SD, proportions were done using contingency tables. The categorical analysis was done with Chi square test and Mc-Newman test. For the quantitative analysis, student $\mathrm{T}$ test (paired \& unpaired) were used. The $\mathrm{p}$ value below 0.05 was considered significant.

All the patients were applied calcaneal traction and advised elevation of the limb, till the day of surgery and were constantly observed for any deterioration in the distal neurovascular status of the limb. Patients with open injuries were taken up immediately for wound debridement and irrigation before the definitive fixation. Prophylactic intravenous cephalosporins and aminoglycosides were administered to all the cases till at least three days after surgery. The condition of the soft tissues, apart from the general condition of the patient, was the most important factor in deciding the time up to surgery. A valid, informed consent for surgery was obtained from the patients and they were taken up for surgery when the swelling of the soft tissue cover showed a reversing trend. Preoperatively, anteroposterior and lateral radiographs of the upper tibia, 3D CT images of the knee, and routine pre-anaesthetic investigations were done. The patients were administered, either general or spinal anaesthesia, as decided by the anaesthetist. No tourniquet was used. In cases of intra-articular extension of fracture line, reduction of articular fragments was done percutaneously with large reduction forceps by close manipulation and traction in the long axis of the limb, under image intensifier.

Limited incisions for minimal open reduction were given to elevate the articular fragments through a small anteromedial cortical window in the metaphyseal region and, in some cases where JESS was used, an additional fixation with 1 or 2 cancellous condylar lag screw was done in cases with articular surface depression of more than $3 \mathrm{~mm}$. Guide pins were inserted after reduction, over which cannulated, $7 \mathrm{~mm}$ partially threaded, lag screws were inserted in the juxta articular region, either one or two in number, so as to engage the major condylar fragments in a lag fashion and also to maintain reduction of articular surface. The screws were not used in cases where the intraarticular fragments were minimally displaced. 


\section{Application of JESS}

Three, long, smooth Kirchner wires of $3 \mathrm{~mm}$ diameter were inserted in the proximal tibial condylar fragment through the safe zones, atleast $1.5 \mathrm{~cm}$ away from the articular surface. These wires were then fixed to head rings, which were half rings by means of link joints after tensioning. These head rings were two in number and concentric, so as to provide the desired stability to the ring assembly of the proximal condylar fragment. Then, 3 Kirchner wires of $3 \mathrm{~mm}$ each were inserted in the tibial shaft from lateral aspect to exit from the medial side. The head ring assembly was then connected to the shaft assembly while giving manual traction to reduce the metaphyseal fragments by ligamentotaxis and aligning it properly without angulation in the sagittal or coronal plane. The assembly was then locked by way of a series of connecting rods and link joints after checking the reduction as well as alignment under the image intensifier, so as to provide a secure, stable and rigid frame.

\section{Application of hybrid external fixator}

Beaded olive wires were inserted through the safe zones, at least $1.5 \mathrm{~cm}$ away from the articular surface. These wires were then connected to Ilizarov semicircular frame of appropriate size by means of link joints after tensioning, thus ensuring a 6-point fixation. The distal assembly consisted of 2 or 3 antero-posterior schanz screws in tibial diaphysis connected by a tubular rod. This tubular rod extended proximally to connect this distal assembly to the Ilizarov semicircular ring in the proximal fragment after ensuring reduction of the fracture fragments by distraction and manipulation and proper alignment. The assembly was hence locked by way of a series of connecting tubular rods and universal clamps, after checking the reduction as well as alignment under image intensifier, so as to provide a secure, stable and rigid frame fixation.

\section{Application of Ilizarov}

Beaded olive wires were inserted through the safe zones, at least $1.5 \mathrm{~cm}$ away from the articular surface. These wires were then connected to Ilizarov frame of appropriate size. Depending on the complexity of the fracture, one to three additional rings were fixed with two to three wires at each level, in the tibial diaphysis and these multiple rings were connected with steel threaded rods. To eliminate the residual displacement of the bone fragments, some additional stabilizing olive wires were inserted at different levels. All the wires were assembled and tensioned to at least $110 \mathrm{~kg}$, as it is marked on the tensioner. From the first postoperative day, patients were put on a physiotherapy regimen consisting of knee mobilization exercises, both passive and active-assisted. Pin tract cleaning with hydrogen peroxide and povidone iodine was done daily. ${ }^{12}$ Touch-down weight bearing was allowed until radiological union was evident. The fracture union was determined using radiographic and clinical assessment at follow up visits in the orthopaedic clinic, scheduled at 6 ,
12 and 24 weeks postoperatively. The patient was looked for complications, if any and functional outcome was assessed using the KSS (Knee Society score) criteria. ${ }^{13}$ External fixator was removed after the patient was advised full weight bearing, on evidence of clinical and radiological union and he was instructed to put on a removable long leg brace while walking, for few days.

\section{RESULTS}

22 patients of AO-41-A3, AO-41C2, AO-41-C3 proximal tibial fractures were admitted in this institute from January 2019 to June 2020. All 22 patients (100\%) were male. Fourteen cases $(64 \%)$ involved the left tibia and eight (36\%) fractures were on right. The mean patient age was 49.5 years (range 26-75). Majority of the patients were in the age group of more than 45 year old. Road traffic collisions accounted for most of the injuries $(n=14)$, while fall from bike $(n=8)$. Compound injury was present in 8 patients $(36.36 \%)$, with 2 having grade II, 2 having grade IIIa and 4 were grade IIIb compound injury according to the Gustilo Anderson's classification of compound fractures. ${ }^{14}$ Associated fracture of the fibula was present in 12 patients $(54.54 \%)$ and/or head injury was present in 2 patients $(9.09 \%)$.

Preoperatively, all the patients had associated soft tissue complications ranging from swelling and bruising of overlying the soft tissue cover to blister formation in 6 cases $(27.27 \%$ ) and compartment syndrome in 2 patients (18.18\%). Fasciotomy was performed in both these patients who had developed compartment syndrome and a knee spanning external fixator was applied in these cases, to aid in temporary fracture reduction and wound observation and dressings, prior to definitive management with a hybrid fixator.

The mean duration between admission and day of surgery was 7 days (range 1-14 days). There were no significant intra operative complications. Blood transfusion was not required for any of the patients during surgery. Tourniquet was not used in any of the patients. The average duration of postoperative stay of the patient was 5 days (range 2-15 days).

The range of motion averaged 115 degrees of flexion (range 90-125 degrees). Neither of the patient showed any significant angulation (more than 5 degree, sagittal or coronal) nor shortening of the limb.

The clinical and functional results were assessed using the parameters of pain, knee range of motion, any flexion contracture or extension lag, varus-valgus alignment and stability in both anteroposterior and mediolateral aspects, using the KSS criteria. In this study mean KSS was 79.04 (range 65-100) in 22 patients. As evaluated by KSS, 8 patients had excellent outcome (score >80), 10 patients had good outcome (score 70-80), 4 patients had fair outcome (score 60-70); and none had poor (score <60) outcome. 
Complication seen was pin tract infection in 2 patients (9.9\%) treated with Ilizarov at 3 months of follow up, which was managed by regular pin site dressings. Another complication was non-union in 1 patient $(4.54 \%)$ of hybrid external fixator at 6 months follow up, which was managed later on by ORIF (plating with bone grafting).

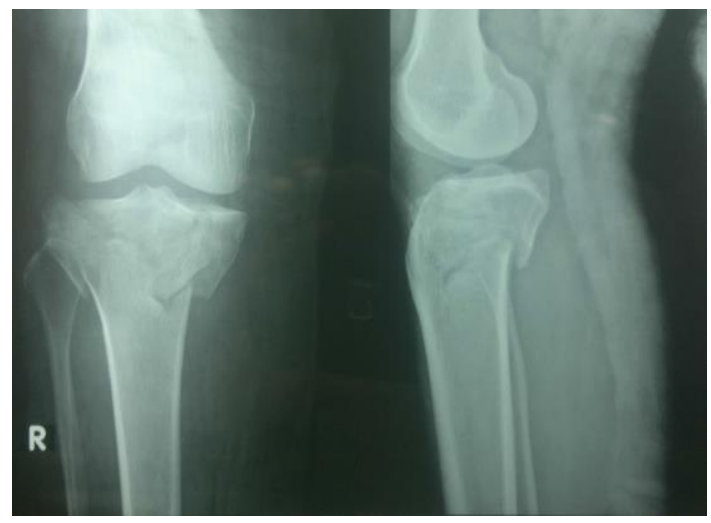

Figure 1: Preoperative X-ray of a patient.

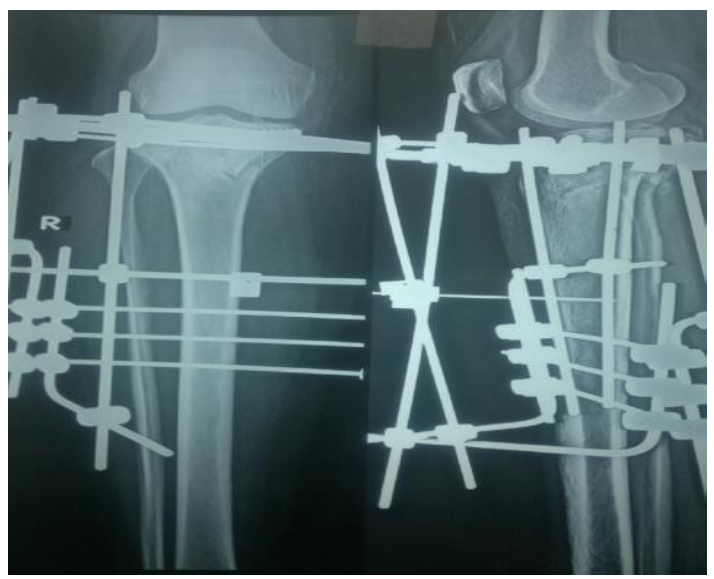

Figure 2: Postoperative X-ray of patient treated with JESS at 6 weeks.

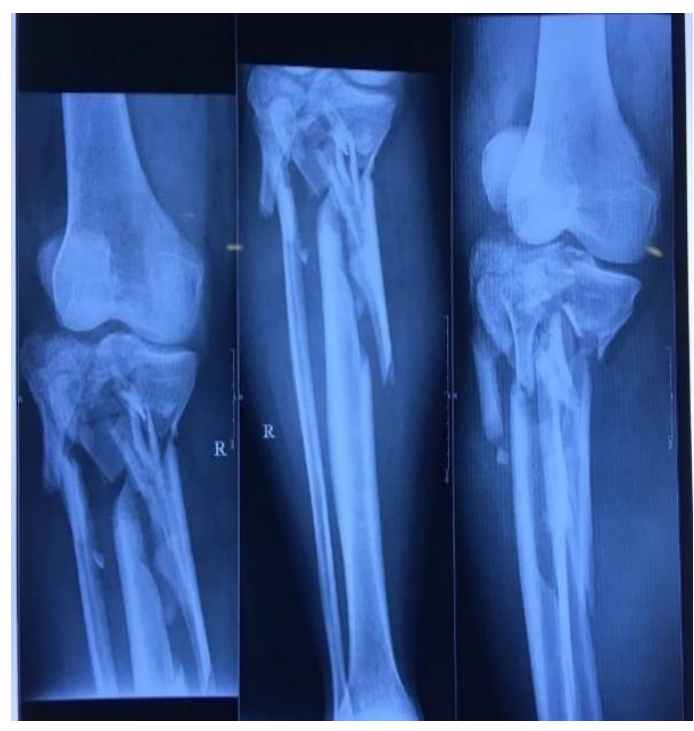

Figure 3: Preoperative X-ray of a patient.

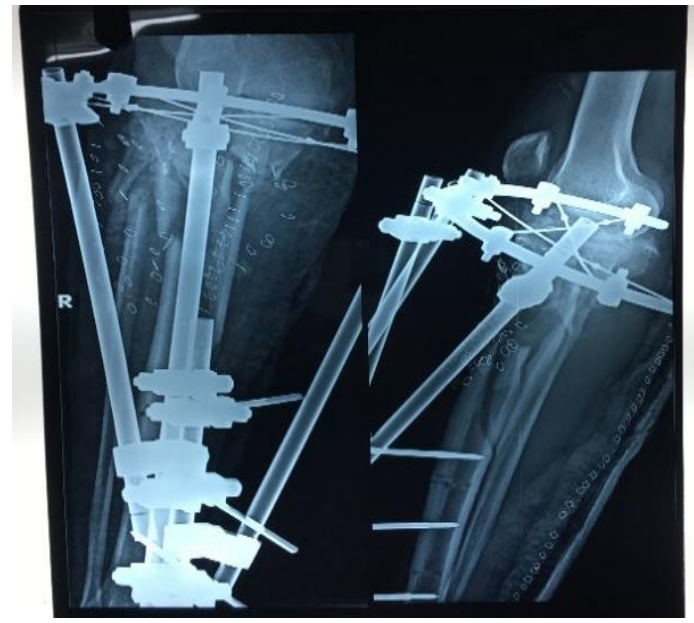

Figure 4: Postoperative X-ray of patient treated with hybrid external fixator.

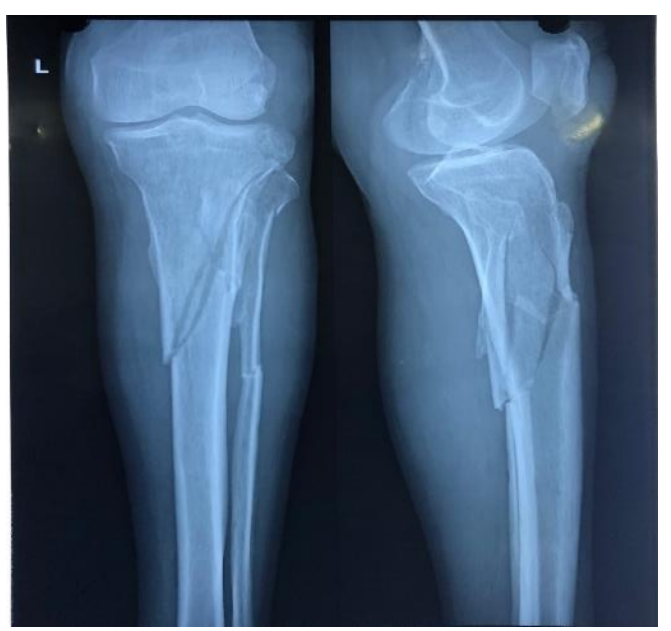

Figure 5: Preoperative X-ray of a patient.

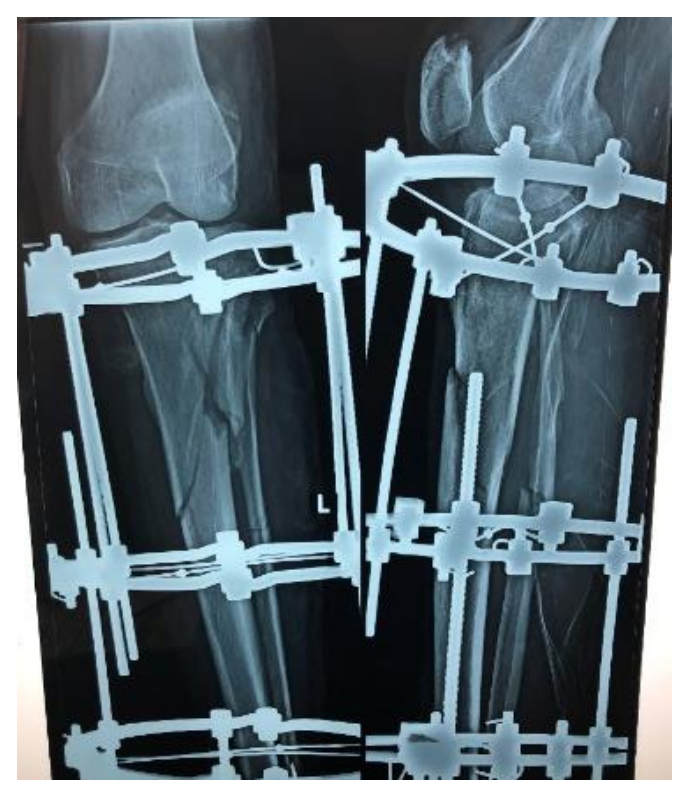

Figure 6: Postoperative X-ray of patient treated with Ilizarov external fixator. 

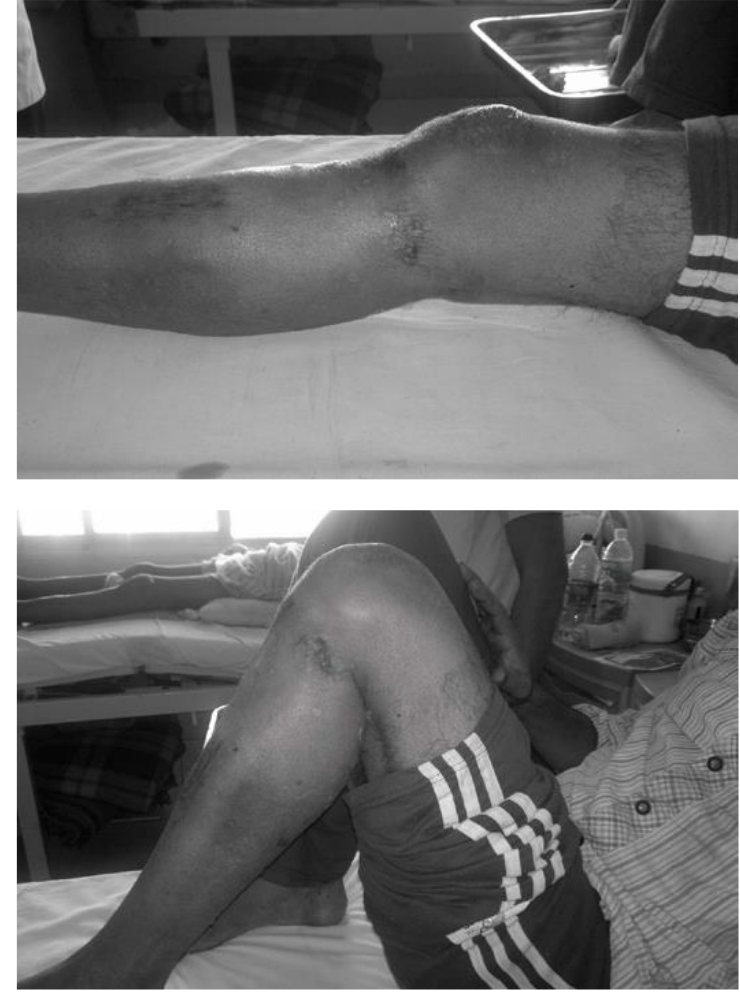

Figure 7: Picture showing range of movement in a patient after removal of JESS at 14 weeks.

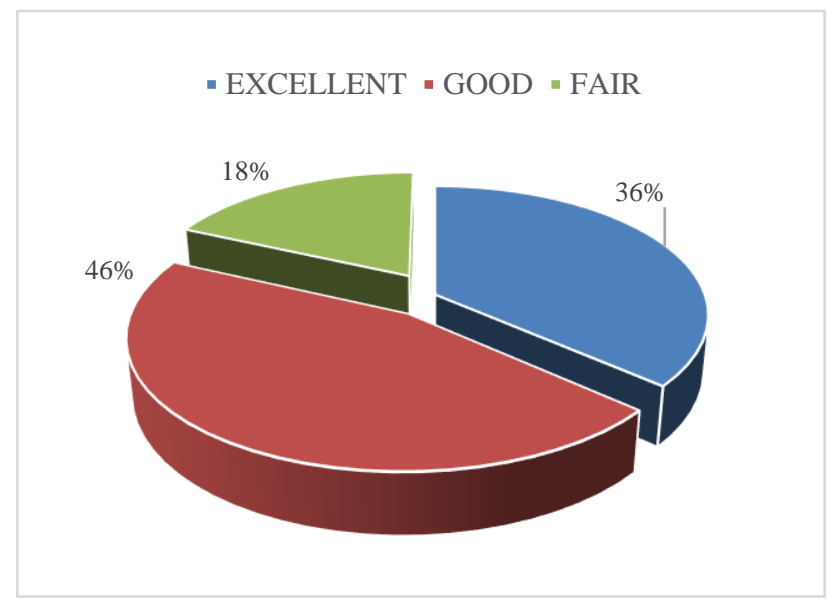

Figure 8: Pie chart showing functional results as percentage at 6 months.

\section{DISCUSSION}

Metaphyseal fractures of the tibia are among the most difficult fractures to treat. The status of the soft tissues, the degree of comminution and articular damage sustained at the time of injury affect the long-term clinical results. The goal of operative treatment is to obtain anatomic realignment of the fractured bones while providing enough stability to allow early motion. This should be accomplished using techniques that minimize osseous and soft tissue devascularization in the hopes of decreasing the complications resulting from treatment. The conventional double plating technique of internal fixation of these fractures involves incisions through the compromised soft tissue cover, extensive soft tissue stripping and hence devitalization of the already traumatized fracture fragments. Consequently, these techniques have often been associated with complications like overlying soft tissue necrosis, problems in wound healing, exposure of metallic hardware and high rate of deep infection including osteomyelitis. ${ }^{15}$ Plating with a relatively newer technique of less invasive stabilization system (LISS) has also been reported to have a higher incidence of postoperative malalignment and symptomatic hardware irritation. ${ }^{16}$ The Canadian Orthopaedic Trauma Association reported lower rate of early postoperative complications and better functional score in patients treated with external rather than internal fixation, for these fractures. ${ }^{17}$ Even in the presence of severe soft tissue compromise, the external stabilization systems, in contrast to traditional techniques of internal fixation, allow for early surgery and hence early stabilization of the fracture with minimal devitalization. ${ }^{18}$

Zeman et al in their study of 19 patients of periarticular fractures of the tibia treated them with hybrid external fixators. ${ }^{8}$ According to the Rasmussen and Bray scoring system, they evaluated the results to be excellent in 5 $(26 \%)$, very good in $6(32 \%)$, fair in $5(26 \%)$ and poor results in 3(16\%). Aggarwal et al in their study of 35 patients using hybrid external fixation for high energy peri articular fractures of the tibia, had results that were good to excellent in $30(86 \%)$, fair in $2(6 \%)$ and poor in $3(8 \%)$ by using knee society scoring system. ${ }^{19}$ Gupta et al conducted a study on management of tibial metaphyseal fractures by hybrid external fixator. ${ }^{20}$ They included 23 patients and they observed that IOWA knee score was excellent in 5 , good in 14, fair in 3 and poor in 1 patient.

Fixation using small tensioned beaded olive wires or $3 \mathrm{~mm}$ $\mathrm{k}$-wires as in JESS prevents further iatrogenic soft tissue injury and minimizes additional devitalization of the bone and avoids disruption of periosteal and endosteal blood supply of the proximal tibia. It offers superior juxtaarticular, metaphyseal purchase, fixes small cancellous fracture fragments, and allows for early range of movement. ${ }^{21}$ This reduces hospital stays and costs. The tensioned olive wires or $\mathrm{k}$-wires provide superior reduction and inter fragmentary compression of metaphyseal fracture components and facilitates fine adjustment of angular and rotational deformity. Where JESS was used, inter-fragmentary compression of the articular surface, wherever needed, was provided with 1 or 2 cancellous condylar screws and/or indirect tensioning of $\mathrm{k}$-wires. In this study, we have used 3 wires proximally, thus giving a six-point fixation to ensure stability. In a study by Watson et al (2002) in each of the 12 patients, only 2 olive wires were used for repair of the articular surface. $^{22}$ At the final follow-up, in 2 of them the correction achieved perioperatively was lost by $3 \mathrm{~mm}$ (depression). Therefore, a 2-wire construct was inadequate for which at least 3 or more out-of-plane wires are recommended. 
We used 10 hybrid external fixators, 8 JESS and 4 Ilizarov fixators. Both the cases of pin track infections were seen where Ilizarov fixator was used and non-union was seen in a case of hybrid external fixator. While satisfactory result was seen in all type of methods of fixation, patients operated with hybrid external fixator had $40 \%$ excellent and $40 \%$ good result, JESS had $75 \%$ excellent and $25 \%$ good results and Ilizarov had 50\% good and 50\% fair result.

Pin tract infection is a potential problem despite the use of small wires. To avoid the disastrous complication of septic arthritis, the small wires were placed extra-articularly, at least $15 \mathrm{~mm}$ away from the articular surface besides this, some of the other disadvantages of the technique was that it needed careful preoperative planning so as to assess the fracture anatomy for appropriate wire placement and also required thorough knowledge of the safe zones for wire placement in the proximal tibia.

The mandate of this study was broad and involved assessing the outcome using circular external fixators without any comparison between the various categories of circular external fixators. Therefore, a further comparative study can be done to assess the outcome in each individual subcategory. However, as a whole, this class of circular external fixators seems to be a promising technique for minimally invasive fixation of metaphyseal fractures of tibia.

\section{CONCLUSION}

Circular external fixation is a promising modality for surgical treatment of comminuted upper metaphyseal tibial fractures. It allows satisfactory realignment of the fractured fragments and their stabilization besides early mobilization of the joints and care of associated soft tissue injuries, without significant complications.

Funding: No funding sources

Conflict of interest: None declared

Ethical approval: The study was approved by the institutional ethics committee

\section{REFERENCES}

1. Egol KA, Tejwani NC, Capla EL, Wolinsky PL, Koval KJ. Staged management of high-energy proximal tibia fractures (OTA types 41): the results of a prospective, standardized protocol. J Orthop Trauma. 2005;19(7):448-55.

2. Gupta A, Anjum R, Singh N, Hackla S. Outcome of distal both bone leg fractures fixed by intramedulary nail for fibula \& MIPPO in tibia. Arch Bone Jt Surg. 2015;3(2):119.

3. Bucholz RW, Court-Brown CM, Heckman JD, Tournetta PMD. Rockwood and Green's Textbook of orthopaedic fractures in adults. 7th ed. USA: Wolters Kluwer; 2003.
4. Young M, Barrack RL. Complications of internal fixation of tibial plateau fractures. Orthop Rev. 1994;23(2):149.

5. Yilmaz E, Belhan O, Karakurt L, Arslan N, Serin E. Mechanical performance of hybrid Ilizarov external fixator in comparison with Ilizarov circular external fixator. CliniC Biomech. 2003;18(6):518-22.

6. Fleming B, Paley D, Kristiansen T, Pope M. A biomechanical analysis of the Ilizarov external fixator. Clinic Orthop Rel Researc. 1989;241:95-105.

7. Babis GC, Evangelopoulos DS, Kontovazenitis P, Nikolopoulos K, Soucacos PN. High energy tibial plateau fractures treated with hybrid external fixation. J Orthop Surg Resear. 2011;6(1):35.

8. Zeman J, Matejka J. Use of a hybrid external fixator for treatment of tibial fractures. Acta Chir Orthop Traumatol. 2005;72(6):337.

9. Zahid M, Sherwani MKA, Siddiqui YS, Abbas M, Asif N, Bin A. The role of the Jess (Joshi's External Stabilization System) fixator in the management of tibial plateau fractures which are associated with severe soft tissue injuris. J Clinic Diagn Researc. 2010;4:3356-61.

10. Hall JA, Beuerlein MJ, McKee MD. Open reduction and internal fixation compared with circular fixator application for bicondylar tibial plateau fractures: surgical technique. J Bone Joint Surg Am. 2009;91:74-88.

11. Subasi M, Kapukaya A, Arslan H, Ozkul E, Cebesoy O. Outcome of open comminuted tibial plateau fractures treated using an external fixator. J Orthop Sci. 2007;12(4):347-53.

12. Babis GC, Evangelopoulos DS, Kontovazenitis P, Nikolopoulos K, SoucacosPN. High energy tibial plateau fractures treated with hybrid external fixation.J Orthop Surg Res. 2011;6:35-41.

13. Insall JN, Dorr LD, Scott RD, Scott WN. Rationale of the Knee Society clinical rating system. Clinic Orthop Relat Res. 1989;(248):13-4.

14. Kim PH, Leopold SS. Gustilo-Anderson Classification. Clin Orhtop Relat. 2012;470(11):3270-4.

15. Ruffolo MR, Gettys FK, Montijo HE, Seymour RB, Karunakar MA. Complications of high-energy bicondylartibial plateau fractures treated with dual plating through 2 incisions. J Orthop Trauma. 2015;29(2):85-90.

16. Jiang R, Luo CF, Wang MC, Yang TY, Zeng BF. Acomparative study of lessinvasive stabilization system (LISS) fixation and two-incision double plating forthe treatment of bicondylartibial plateau fractures. Knee. 2008;15(2):139-43.

17. Canadian Orthopaedic Trauma Society. Open reduction and internal fixation compared with circular fixator application for bicondylartibial plateau fractures. Results of a multicenter, prospective, randomized clinical trial. J Bone Joint Surg Am. 2006;88(12):2613-23.

18. Hall JA, Beuerlein MJ, McKee MD. Open reduction and internal fixation compared with circular fixator 
application for bicondylartibial plateau fractures. Surgical technique. J Bone Joint Surg Am. 2009;91:74-88.

19. Aggarwal AK, Nagi ON. Hybrid external fixation in periarticular tibial fractures. Good final outcome in 56 patients. Acta Orthop Belg. 2006;72(4):434-40.

20. Gupta SV, Sunil G. Management of tibial metaphyseal fractures by hybrid external fixator. Open J Orthop. 2014;4(3):84-9.
21. Kataria H, Sharma N, Kanojia RK. Small wire external fixation for high-energy tibial plateau fractures. J Orthop Surg. 2007;15(2):137-43.

22. Watson JT, Coufal C. Treatment of complex lateral plateau fractures using Ilizarov techniques. Clinic Orthop Rel Res. 1998;353:97-106.

Cite this article as: Patel $\mathrm{P}$, Singh $\mathrm{H}$, Agarwal KK, Patel T, Saoji K, Patel D. Functional outcome of comminuted upper metaphyseal tibial fracture treated with circular external fixators. Int J Res Orthop 2021;7:622-8. 